The medical emergency plan designed for this stadium will be described, including what events can trigger the plan and who can activate it. The stadium layout will show the locations of the central medical post and satellite posts. Communication protocols, lists of stored drugs, and medical team composition and location during matches will be discussed. Cardiac problems are among the most common major emergencies during this kind of event; the number and placement of defibrillators will be shown. Also presented are the number and location of rescue vehicles, together with means of evacuation.

Data from a year of operation will be presented, including the number and type of events, actions taken, and lessons learned. Factors such as weather, risk, and importance of the game will be discussed. It is shown that bad weather increases orthopedic injuries and that matches between rival teams generally result in more emergency cases. The analysis of this one-year experience led to minor changes in the emergency planning that also will be described.

The emergency plan responds efficiently to the prehospital needs for events of such type and is integrated well with the Portuguese Emergency System.

Keywords: Dragon Stadium; football; medical emergency plan; Portugal; soccer

Prebosp Disast Med 2005;20(2):s40-41

\section{Hellenic National Centre for Emergency Care's Preparedness for the Olympic Games in Athens 2004 A. Zygoura}

Hellenic National Centre for Emergency Care (HNCEC), Greece

Greece, the birthplace of the Olympic Games, was designated to host the 28th Olympiad in Athens during the summer of 2004.

To ensure the success of the 28th Olympiad in Athens, the Hellenic National Centre for Emergency Care (HNCEC) had to plan for providing emergency prehospital health care and transportation for all the people that would visit the Olympic and Paralympics Venues in Athens and other Olympic cities.

To respond to all these needs, the HNCEC appointed a Committee for Operational Planning to edit and perform the plan for providing health care during the Olympic Games. Health services provided included conventional health care but also mass-casualty health care related to natural disasters, transportation crashes, terrorist attacks, and chemical, biological, radiological, and nuclear CBRN threats.

The Committee faced a challenge in planning for an adequate response and for the acquisition of special equipment, ambulances, a new command and coordination center, insertion of new communication systems (tetra, AVL, GPS), enforcement and training of HNCEC's personnel, special plans for mass-casualty, healthcare-related disasters, terrorist attacks, and CBRN threats. All plans had to respect interagency cooperation, crisis communication, and other security issues. For the coverage of all athletic venues, "Olympic Village" Olympic Family hotels, VIPs and the media centers, 100 ambulances were deployed, along with 13 mobile intensive care units (ICUs), and five emergency medical service motorcycles. For the coverage of the rest of the city, 100 ambulances, ten mobile ICUs, and six emergency medical services (EMS) motorcycles were deployed. During the Olympic Games in Athens, 413 persons had to be treated and transported from the Olympic Venues to the Olympic Hospitals or the Olympic Village Polyclinic, while from the other Olympic cities, 34 persons had to be treated and transported.

The 28th Olympiad in Athens was successful, and all the international media reported the same. The Greek health services fulfilled the requirements of the organization, contributing to the success of the games.

Keywords: Athens; emergency medical services; Greece; healthcare Hellenic National Centre for Emergency Care (HNCEC); Olympics; planning

Prebosp Disast Med 2005;20(2):s4

\section{Modeling Mass Gatherings and the Development of Theory \\ Paul Arbon}

The University of Canberra and the Canberra Hospital, Australia

Mass gatherings are an increasingly common feature of modern society, and their impact on the demand for health services has been documented widely. However, descriptive papers that focus on a single event or event type dominate the literature, and, while these contribute to our understanding of the patient care required at such events, they do not provide an adequate analysis of the health effects of the mass-gathering phenomenon itself. This presentation argues for the development of conceptual models and theories to underpin mass-gathering research. The development of theory and conceptual models provides a strategy to improve the understanding of the health effects of mass gatherings across different contexts and situations. Two preliminary conceptual models are presented as a means to encourage further debate about the dominant influences on the health of people where crowds gather, and to promote less superficial forms of analysis of the research data.

These conceptual models are based on the idea that mass-gathering health can be understood as an inter-relationship between three domains: (1) the biophysical; (2) the environmental; and (3) the psychosocial. Key features that influence the rate of injury and illness characterize each domain. These key features are well understood and combine to produce an effect-the patient presentation rate, and a response-the health plan. A new element, the latent potential for injury and illness, is introduced as a mechanism for describing a precursor state important in assessing health risk during mass gatherings.

Keywords: conceptual models; health risk; mass gatherings; theories Prebosp Disast Med 2005;20(2):s41 